\title{
Optimal Power Flow using Cockroach Swarm Optimization
}

\author{
M. A. El-dosuky \\ Computer Science Department,Mansoura University \\ Faculty of Computer and Info \\ Mansoura University, Egypt. P.O. 35516
}

\begin{abstract}
Optimal Power Flow(OPF) faces challenges of convergence and local minima. This paper reviews nomenclature and literature of both OPF and metaphor-based heuristic optimization, emphasizing Cockroach Swarm Optimization (CSO). Then the paper investigates CSO as applied to OPF. The solution is tested on the benchmark systems such as IEEE 9 bus system using Matpower.
\end{abstract}

\section{General Terms}

Global optimization, Power Systems

\section{Keywords}

Optimal Power Flow,Cockroach Swarm Optimization

\section{INTRODUCTION}

Optimal Power Flow (OPF) is implicated in both security [49] and economy [13] of operation. Solving an OPF faces many challenges [50] such as convergence [29] and local minima [42] .

The rest of this paper presents OPF nomenclature and metaphorbased heuristic optimization, emphasizing Cockroach Swarm Optimization (CSO), in the second section, before reviewing OPF literature in the third section. Then the paper investigates CSO as applied to OPF in the evaluation section where CSO is tested on benchmark systems, before concluding and showing future directions in the last sections.

\section{BACKGROUND}

\subsection{OPF Formulation}

Power flows can be calculated from voltage phase angle denoted as $\delta_{i}$ [53]. Hence, $v_{i}$, the voltage at node $i$, is

$$
\left|v_{i}\right| \angle \delta_{i}
$$

Admittance matrix $Y$ of size $n_{b} \times n_{b}$ can perfectly describe a network of $n_{b}$ buses [53, 23].

Now, $p_{f}$, the active power injected into bus $f$ at time $t$, is

$$
\sum_{t=1}^{n_{b}}\left|y_{f t} v_{f} v_{t}\right| \cos \left(\delta_{f}-\delta_{t}-\theta_{f t}\right)
$$

and the reactive power, $q_{f}$, is

$$
\sum_{t=1}^{n_{b}}\left|y_{f t} v_{f} v_{t}\right| \sin \left(\delta_{f}-\delta_{t}-\theta_{f t}\right)
$$

where $\theta_{\text {shift }}$ is the phase shift angle.

OPF is a global minimization problem [32, §18], i.e.

$$
\min _{x} f(x)
$$

with constraints :

$$
p_{g}^{f}-p_{d}^{f}=p^{f}
$$

and

$$
q_{g}^{f}-q_{d}^{f}=q^{f},
$$

where $g$ is generation

$$
g(x)=0
$$

and $d$ is demand

OPF operates near maximum loadability limit (MLL), formulated as [30]

$$
\max _{z} \alpha
$$

subject to

$$
\begin{aligned}
& P_{d}=P_{d}^{0}+\alpha d \\
& Q_{d}=\operatorname{diag}\left(\tan \phi^{0}\right) P_{d} \\
& g(z)=0 \\
& z_{\min } \leq z \leq z_{\max }
\end{aligned}
$$

where

$$
z=\left[\begin{array}{ll}
x^{T} & \alpha
\end{array}\right]^{T}=\left[\begin{array}{c}
\Theta \\
V_{m} \\
P_{g} \\
Q_{g} \\
\alpha
\end{array}\right],
$$

Differences among MLL and OPF regarding voltage constraints include [12]:

(1) Magnitudes at the reference buses are locked at their set point values.

(2) Magnitudes at all PV and PQ buses are not constrained from below. 
(3) PQ magnitude upper bounds are relaxed.

(4) PV magnitude upper bounds are set to the voltage set point of correspondent generators.

\subsection{Metaphor-based Heuristic optimization}

Figure 1 depicts an arbitrary search space with many local mini$\mathrm{ma} / \mathrm{maxima}$. This nonlinear problem, and the like, requires global optimization [52, 7, 16].

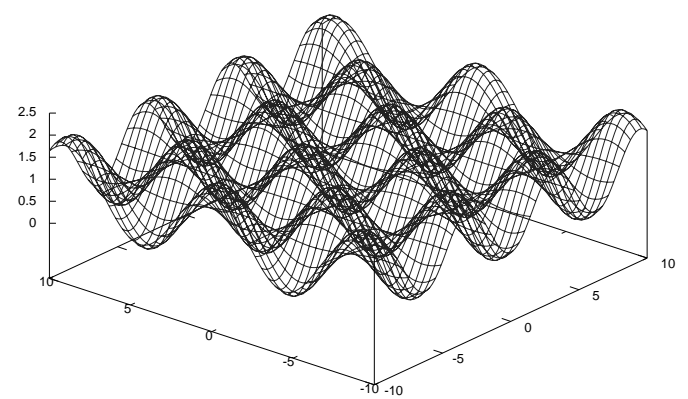

Fig. 1. an arbitrary search space with many local minima/maxima.

Global heuristic optimization, a.k.a metaheuristics [22], scrutinize search spaces using a dichotomy of intensification and diversification [21, 10] .

Metaphor-based optimization [1] usually mimic nature [54] such as Genetic Algorithms (GAs) [24], Cuckoo Search (CS) [55], Hoopoe Heuristic(HH) [19], or swarms [34] of particles(PSO) [35], bees (BCO) [33], or ants (ACO) [18].

\subsection{Cockroach Swarm Optimization (CSO)}

Many algorithms mimic cockroaches [57, 27]. CSO uses:

(1) Swarming of a quorum of cockroaches, excreting a fecal matter trail in the way for the others to follow [5].

(2) Dispersion in presence of danger or resource sparsity [5].

(3) Eliminating each other in resource shortage [57|

Blattodea insects, comprising cockroaches, have an affinity with Hymenoptera,comprising ants and bees, showing gregarious behaviour [40]. Ants are eusocial with distinguishable behavioral specializations [28]. Otherwise, the nocturnal cockroaches swarm in aggregations [9] for collective decision-making [39].

As depicted in figure 2 cockroaches quorum is initialized randomly, as shown in figure 3 Then, they are evaluated, the best individual being associated with a given position in their field of vision. Hence, forming a cluster based on that, as shown in figure 4 CSO converses rapidly as shown in figure 5

\section{PREVIOUS WORK}

Since the original formulation of OPF [13], the Newton-Raphson was the de facto solving method [51, 45] even for ill-conditioned systems [31].

OPF is periodically surveyed [26, 29, 11]. A honorable mention is use of linear programming (LP) [4].

Metaphor-based heuristic optimizations [14, 37] are used such as

-PSO [47, 2] and fuzzy version [8]

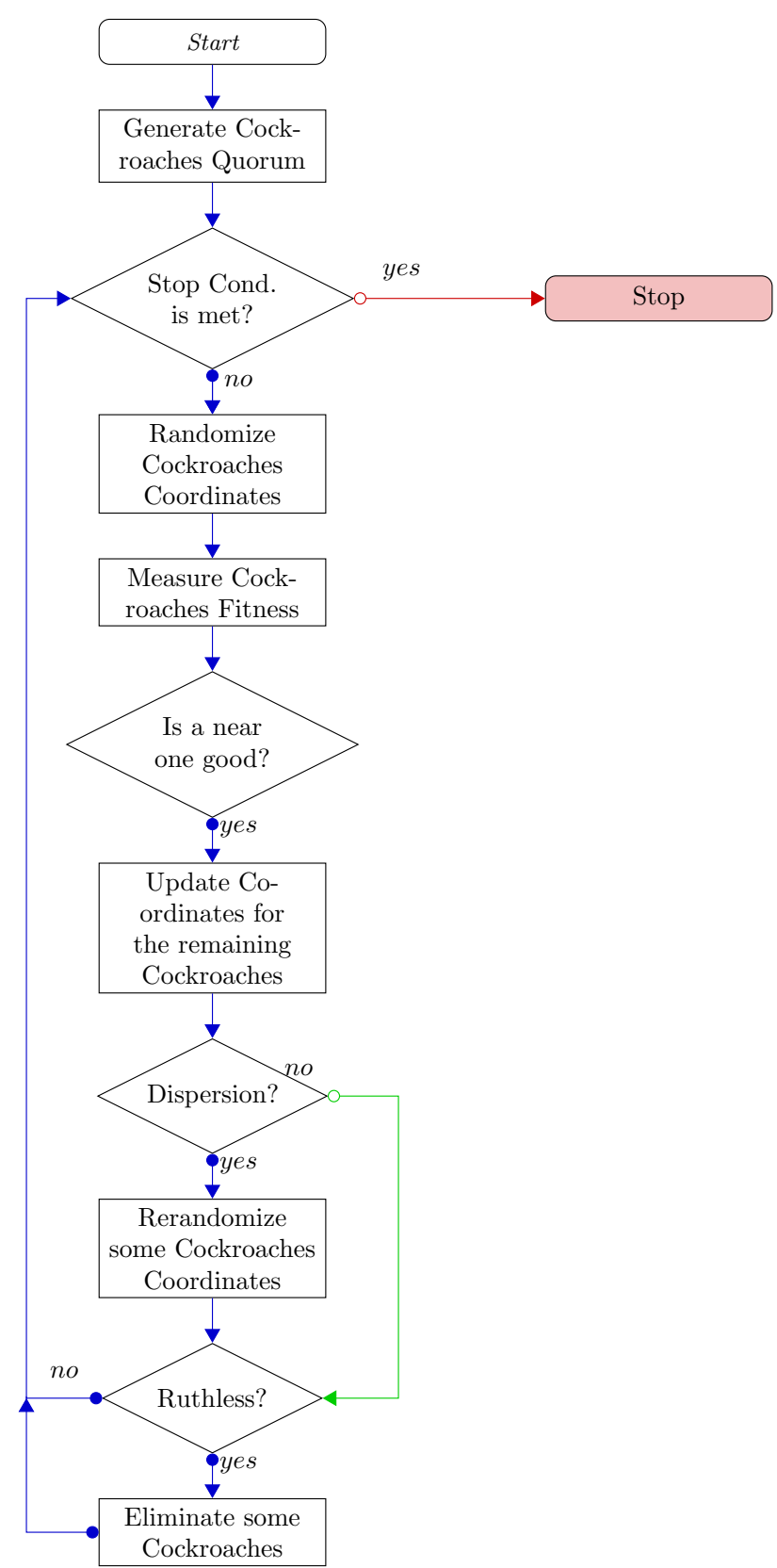

Fig. 2. CSO Flowchart.

\section{-GAs [36, 56] \\ $-\mathrm{ACO}[38$ \\ $-\mathrm{BCO}[48$}

$-\mathrm{CS}[46]$ 


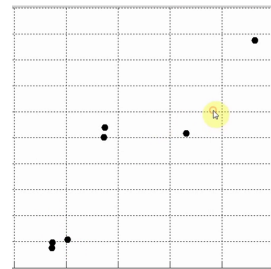

Fig. 3. Initial settings

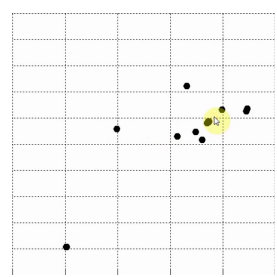

Fig. 4. Cockroach Clustering.

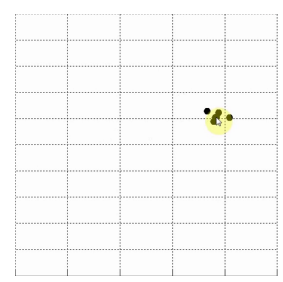

Fig. 5. Pre-Convergence.

\section{EVALUATION}

CSO applied to OPF is tested on the IEEE 9 bus system [6] implemented in MATPOWER.V.6[58] as CASE09.m as shown in figure6 . CASE09 has 1 area, 9 buses, 9 branches, 3 generators and 3 loads. Consider continuation flow, runcpf in MATPOWER.V.6, with no reactive power limits.

$\begin{array}{ccc}\text { Bus } & \text { Volt.magnitude } & \text { Volt.angle } \\ 1 & 1 & 0 \\ 2 & 1 & -50.45 \\ 3 & 1 & -55.24 \\ 4 & 0.84 & -19.34 \\ 5 & 0.77 & -37.63 \\ 6 & 0.82 & -58.71 \\ 7 & 0.61 & -79.87 \\ 8 & 0.78 & -57.92 \\ 9 & 0.74 & -38.42\end{array}$

The results are:

$\begin{array}{ccc}\text { Bus } & \text { P } & \text { Q } \\ -1 & 482 & 362 \\ 2 & 163 & 355.5 \\ 3 & 85 & 305.5 \\ - & & \\ \text { Total: } & 730 & 1023\end{array}$

Compare This with MLL as shown below.
Bus
MLL Load
Voltage

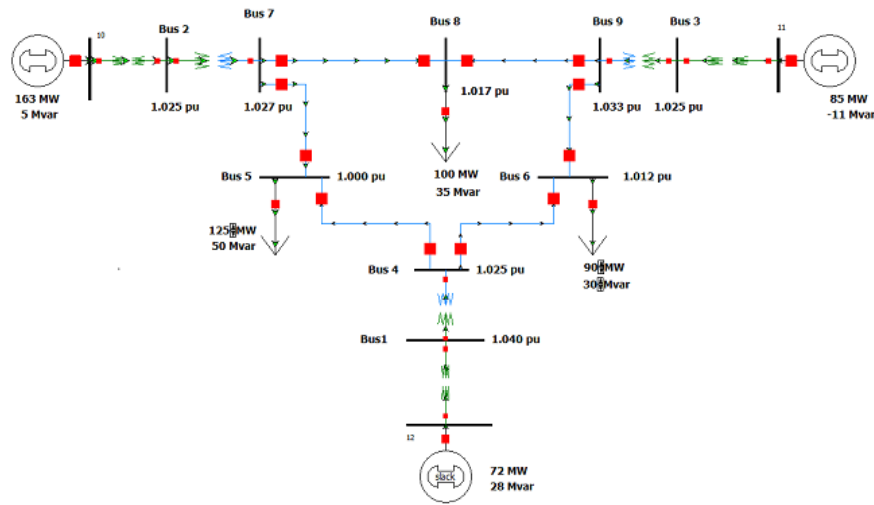

Fig. 6. IEEE 9 bus system [6].

\begin{tabular}{|c|c|c|}
\hline 1 & 0 & 1 \\
\hline 2 & 0 & 1 \\
\hline 3 & 0 & 1 \\
\hline 4 & 0 & 0.87 \\
\hline 5 & 90 & 0.81 \\
\hline 6 & 0 & 0.85 \\
\hline 7 & 439.53 & 0.66 \\
\hline 8 & 0 & 0.82 \\
\hline 9 & 125 & 0.78 \\
\hline
\end{tabular}

All busses have direction 0 , except the 7th has direction 1 . The Reactive power production and generators voltages are :

$\begin{array}{ccc}\text { Bus } & \text { Qgen } & \text { Qmax } \\ 1 & 305.15 & 9999 \\ 2 & 300 & 300 \\ 3 & 255.64 & 300\end{array}$

All with Qmin $=-300, \mathrm{Vm}=\mathrm{Vref}=1$.

CSO converges in 4 iterations, approx.0.29 seconds, giving

$\begin{array}{ccc}\text { Bus } & \text { Volt.magnitude } & \text { Volt.angle } \\ 1 & 1 & 0 \\ 2 & 1 & 9.67 \\ 3 & 1 & 4.77 \\ 4 & 0.99 & -2.41 \\ 5 & 0.98 & -4.02 \\ 6 & 1 & 1.92 \\ 7 & 0.99 & 0.62 \\ 8 & 0.99 & 3.8 \\ 9 & 0.96 & -4.35\end{array}$

The generations are

$\begin{array}{ccc}\text { Bus } & \text { P } & \text { Q } \\ ---1 & 71.95 & 24.07 \\ 2 & 163 & 14.46 \\ 3 & 85 & -3.65 \\ ----1 & 34.88\end{array}$




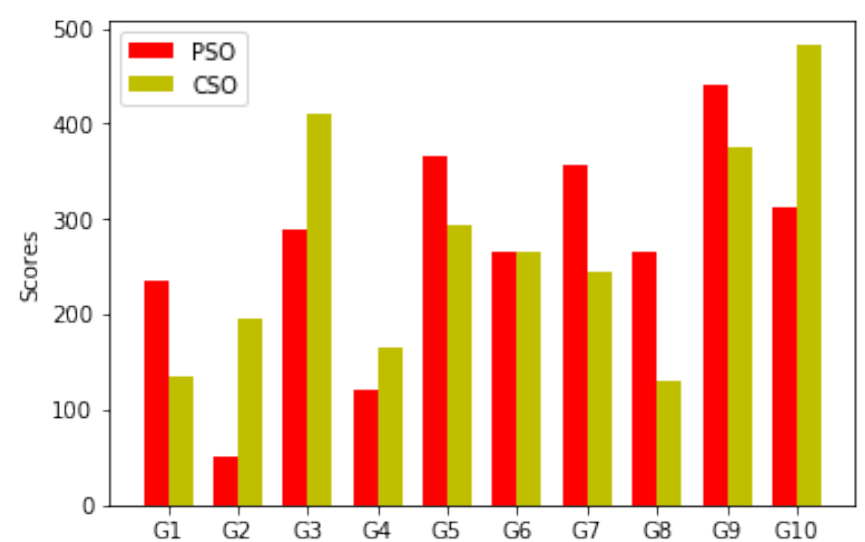

Fig. 7. PSO vs CSO on Systems with 10 groups [15].

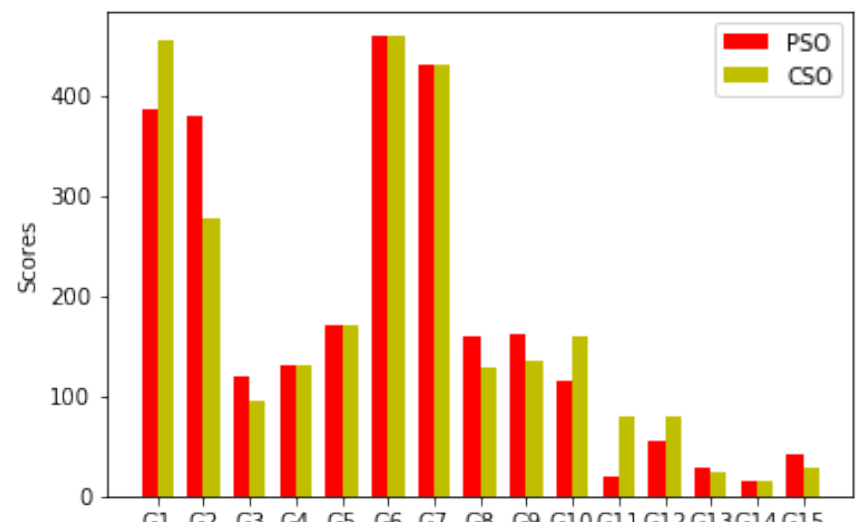

Fig. 8. PSO vs CSO on Systems with 15 groups [20].

As PSO is widely applied in power systems [17, 3], let us compare it with CSO on benchmark systems such as [15] in figure7] and [20]in figure8

\section{CONCLUSION}

CSO shows a fast convergence as compared to PSO.

\section{FUTURE WORK}

An enunciated future work may be applying CSO in other cases such as IEEE Reliability Test test [25] in figure9] to investigate the invariants among systems of varying sizes.

Another direction may be considering improved versions of CSO [43. 44], or parameter optimization [41].

\section{REFERENCES}

[1] Ec bestiary website. https://github. com/f campelo/EC-bestiary

[2] M A Abido. Optimal power flow using particle swarm optimization. International Journal of Electrical Power and Energy Systems, 24(7):563-571, 2002.

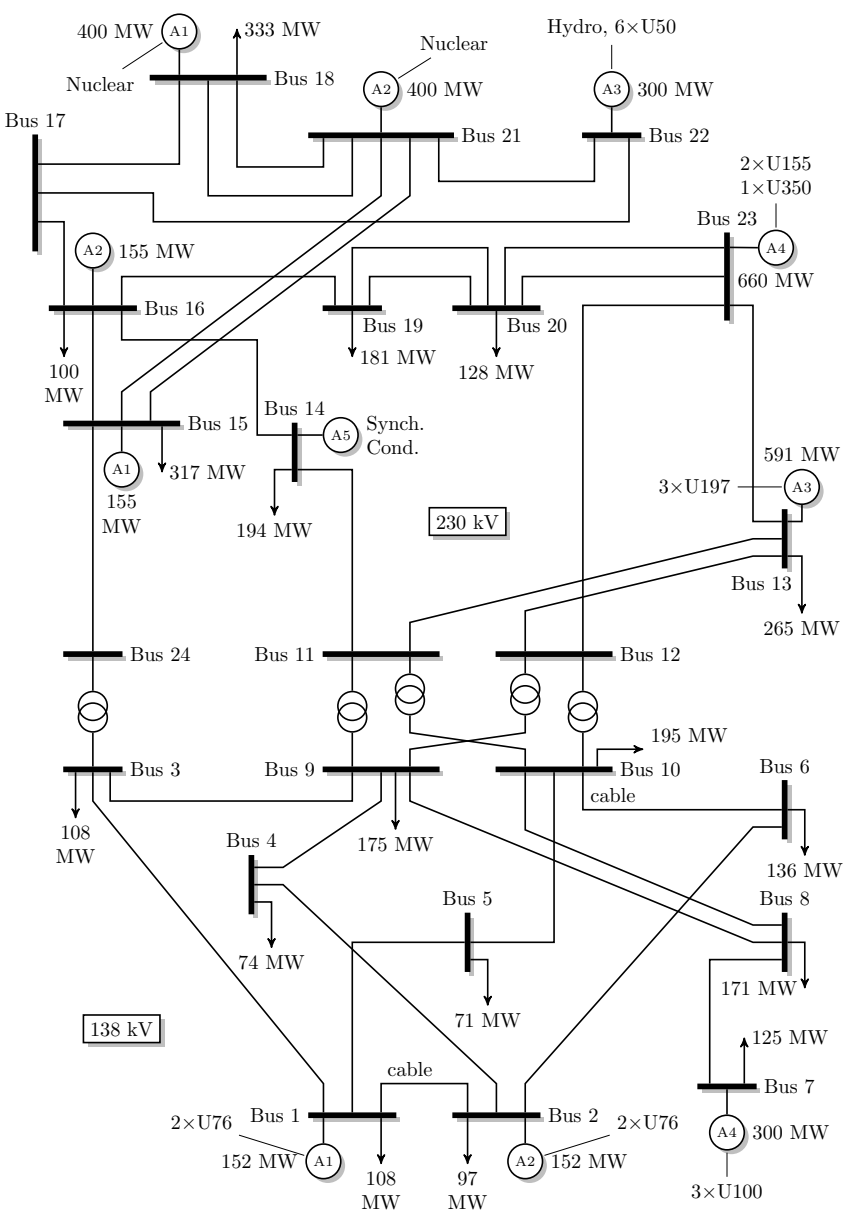

Fig. 9. IEEE Reliability Test test [25].

[3] Mohammed R AlRashidi and Mohamed E El-Hawary. A survey of particle swarm optimization applications in electric power systems. IEEE transactions on evolutionary computation, 13(4):913-918, 2009.

[4] O Alsac, J Bright, M Prais, and B Stott. Further developments in lp-based optimal power flow. IEEE Transactions on Power Systems, 5(3):697-711, 1990.

[5] Jean-Marc Amé, José Halloy, Colette Rivault, Claire Detrain, and Jean Louis Deneubourg. Collegial decision making based on social amplification leads to optimal group formation. Proceedings of the National Academy of Sciences, 103(15):58355840, 2006.

[6] Paul M Anderson and Aziz A Fouad. Power system control and stability. John Wiley \& Sons, 2008.

[7] JS Arora. Introduction to optimum design. 1989.

[8] Mostafa Asghari and Jamshid Mohammadi. Fuzzy multiobjective opf considering voltage security and fuel emission minimization. In Power and Energy Engineering Conference

y. (APPEEC), 2012 Asia-Pacific, pages 1-6. IEEE, 2012.

[9] William J Bell, Louis M Roth, and Christine A Nalepa. Cockroaches: ecology, behavior, and natural history. JHU Press, 2007. 
[10] Christian Blum and Andrea Roli. Metaheuristics in combinatorial optimization: Overview and conceptual comparison. ACM Computing Surveys (CSUR), 35(3):268-308, 2003.

[11] Mary B Cain, Richard P O'neill, and Anya Castillo. History of optimal power flow and formulations. Federal Energy Regulatory Commission, pages 1-36, 2012.

[12] Claudio Cañizares, William Rosehart, Alberto Berizzi, and Cristian Bovo. Comparison of voltage security constrained optimal power flow techniques. In Power Engineering Society Summer Meeting, 2001, volume 3, pages 1680-1685. IEEE, 2001.

[13] J Carpentier. Contribution á l'étude du dispatching économique. Bulletin de la Société Française des Électriciens, 3(1):431-447, 1962.

[14] Jinyin Chen and Dongyong Yang. Optimal power flow optimization based on bio-inspired computing. In Communications and Mobile Computing (CMC), 2010 International Conference on, volume 1, pages 195-199. IEEE, 2010.

[15] Chao-Lung Chiang. Improved genetic algorithm for power economic dispatch of units with valve-point effects and multiple fuels. IEEE transactions on power systems, 20(4):16901699, 2005.

[16] Kalyanmoy Deb. Optimization for engineering design: Algorithms and examples. PHI Learning Pvt. Ltd., 2012.

[17] Yamille Del Valle, Ganesh Kumar Venayagamoorthy, Salman Mohagheghi, Jean-Carlos Hernandez, and Ronald G Harley. Particle swarm optimization: basic concepts, variants and applications in power systems. IEEE Transactions on evolutionary computation, 12(2):171-195, 2008.

[18] Marco Dorigo. Optimization, learning and natural algorithms. Ph. D. Thesis, Politecnico di Milano, Italy, 1992.

[19] M. A. El-Dosuky, M. Z. Rashad, and T. T. Hamza. New hoopoe heuristic optimization. International Journal of Science and Advanced Technology, 2(9):85-90, 2012.

[20] Zwe-Lee Gaing. Particle swarm optimization to solving the economic dispatch considering the generator constraints. IEEE transactions on power systems, 18(3):1187-1195, 2003.

[21] Veysel Gazi and Kevin M Passino. Stability analysis of social foraging swarms. IEEE Transactions on Systems, Man, and Cybernetics, Part B (Cybernetics), 34(1):539-557, 2004.

[22] Fred Glover. Future paths for integer programming and links to artificial intelligence. Computers \& operations research, 13(5):533-549, 1986.

[23] J. Duncan Glover and Mulukutla S. Sarma. Power System Analysis and Design. Brooks/Cole Publishing Co., Pacific Grove, CA, USA, 3rd edition, 2001.

[24] David E Goldberg. Genetic algorithms in search, optimization, and machine learning, 1989. Addison-Wesley, 1989.

[25] Cliff Grigg, Peter Wong, Paul Albrecht, Ron Allan, Murty Bhavaraju, Roy Billinton, Quan Chen, Clement Fong, Suheil Haddad, Sastry Kuruganty, et al. The ieee reliability test system-1996. a report prepared by the reliability test system task force of the application of probability methods subcommittee. IEEE Transactions on power systems, 14(3):1010$1020,1999$.

[26] HH Happ. Optimal power dispatch-a comprehensive survey. IEEE Transactions on Power Apparatus and Systems, 96(3):841-854, 1977.
[27] Timothy C Havens, Christopher J Spain, Nathan G Salmon, and James M Keller. Roach infestation optimization. In Swarm Intelligence Symposium, 2008. SIS 2008. IEEE, pages 1-7. IEEE, 2008.

[28] B Hölldobler and EO Wilson. The ants cambridge. MA: The Belknap Press, Harvard University, 1990.

[29] M Huneault and FD Galiana. A survey of the optimal power flow literature. IEEE transactions on Power Systems, 6(2):762-770, 1991

[30] GD Irisarri, X Wang, J Tong, and S Mokhtari. Maximum loadability of power systems using interior point nonlinear optimization method. IEEE transactions on Power Systems, 12(1):162-172, 1997.

[31] S Iwamoto and Y Tamura. A load flow calculation method for ill-conditioned power systems. IEEE transactions on power apparatus and systems, (4):1736-1743, 1981.

[32] J. Kallrath, P.M. Pardalos, S. Rebennack, and M. Scheidt. Optimization in the Energy Industry. Springer, 2009.

[33] Dervis Karaboga. An idea based on honey bee swarm for numerical optimization. Technical report, Technical report-tr06, Erciyes university, engineering faculty, computer engineering department, 2005.

[34] James Kennedy. Swarm intelligence. In Handbook of natureinspired and innovative computing, pages 187-219. Springer, 2006.

[35] R Kennedy. J. and eberhart, particle swarm optimization. In Proceedings of IEEE International Conference on Neural Networks IV, pages, volume 1000, 1995.

[36] R Kumar et al. Economic power dispatch using evolutionary algorithm. International Journal of Machine Learning and Computing, 2(4):365-370, 2006.

[37] Kwang Y Lee and Mohamed A El-Sharkawi. Modern heuristic optimization techniques: theory and applications to power systems, volume 39. John Wiley \& Sons, 2008.

[38] K Lenin and MR Mohan. Ant colony search algorithm for optimal reactive power optimization. Serbian journal of electrical engineering, 3(1):77-88, 2006.

[39] Mathieu Lihoreau, JT Costa, and Colette Rivault. The social biology of domiciliary cockroaches: colony structure, kin recognition and collective decisions. Insectes Sociaux, 59(4):445-452, 2012.

[40] Mathieu Lihoreau, Jean-Louis Deneubourg, and Colette Rivault. Collective foraging decision in a gregarious insect. Behavioral Ecology and Sociobiology, 64(10):1577-1587, 2010.

[41] Mohamed Arezki Mellal and Edward J Williams. Parameter optimization of advanced machining processes using cuckoo optimization algorithm and hoopoe heuristic. Journal of Intelligent Manufacturing, 27(5):927-942, 2016.

[42] JA Momoh, RJ Koessler, MS Bond, B Stott, D Sun, A Papalexopoulos, and P Ristanovic. Challenges to optimal power flow. IEEE Transactions on Power Systems, 12(1):444-455, 1997.

[43] Ibidun Christiana Obagbuwa and Ademola Philips Abidoye. Binary cockroach swarm optimization for combinatorial optimization problem. Algorithms, 9(3):59, 2016.

[44] IC Obagbuwa and AO Adewumi. An improved cockroach swarm optimization. The Scientific World Journal, 2014, 2014. 
[45] John Peschon, Dean S Piercy, William F Tinney, Odd J Tveit, and Michael Cuenod. Optimum control of reactive power flow. IEEE Transactions on Power Apparatus and Systems, (1):40-48, 1968.

[46] M RamaMohana Rao and AV Naresh Babu. Optimal power flow using cuckoo optimization algorithm. International Journal of Advanced Research in Electrical, Electronics and Instrumentation Engineering, 2(9):4213-18, 2013.

[47] T Saravanan, G Saritha, and V Srinivasan. Optimal power flow using particle swarm optimization. Middle-East Journal of Scientific Research, 20(11):554-1560, 2014.

[48] Linda Slimani and Tarek Bouktir. Economic power dispatch of power systems with pollution control using artificial bee colony optimization. Turkish Journal of Electrical Engineering \& Computer Sciences, 21(6):1515-1527, 2013.

[49] Brian Stott, Ongun Alsac, and Alcir J Monticelli. Security analysis and optimization. Proceedings of the IEEE, 75(12):1623-1644, 1987.

[50] WF Tinney, JM Bright, KD Demaree, and BA Hughes. Some deficiencies in optimal power flow. IEEE Transactions on Power Systems, 3(2):676-683, 1988.

[51] William F Tinney and Clifford E Hart. Power flow solution by newton's method. IEEE Transactions on Power Apparatus and systems, (11):1449-1460, 1967.

[52] Thomas Weise. Global optimization algorithms-theory and application. Self-published, 2, 2009.

[53] Allen J. Wood and Bruce F. Wollenberg. Power Generation Operation and Control. Wiley, New York, New York, second edition, 1996.

[54] Xin-She Yang. Nature-inspired metaheuristic algorithms. Luniver press, 2010.

[55] Xin-She Yang and Suash Deb. Cuckoo search via lévy flights. In Nature \& Biologically Inspired Computing, 2009. NaBIC 2009. World Congress on, pages 210-214. IEEE, 2009.

[56] Mimoun Younes and Mostafa Rahli. Economic power dispatch using the combination of two genetic algorithms. $I U$ Journal of Electrical \& Electronics Engineering, 6(2):175181, 2006

[57] Chen ZhaoHui and Tang HaiYan. Cockroach swarm optimization. In 2010 2nd International Conference on Computer Engineering and Technology, 2010.

[58] R.D. Zimmerman, C.E. Murillo-Sanchez, and R.J. Thomas. MATPOWER: steady-state operations, planning and analysis tools for power systems research and education. Power Systems, IEEE Transactions on, 26(1):12-19, February 2011. 